\title{
The Effect of Sprint and Endurance Training on Electromyogram Signal Analysis by WAVELETS
}

\author{
au2 Cora Huber, ${ }^{1}$ Beat Göpfert, ${ }^{1}$ Patrick Kugler, ${ }^{2,3}$ and Vinzenz von Tscharner ${ }^{2}$ \\ ${ }^{1}$ Laboratory of Biomechanics and Biocalorimetry $\left(L O B^{2}\right)$, University of Basel, Basel, Szwitzerland; ${ }^{2}$ Human Performance \\ Laboratory, Faculty of Kinesiology, The University of Calgary, Calgary, Alta, Canada; and ${ }^{3}$ Department of Computer Science, \\ AU3 University of Erlangen-Nuremberg, Germany
}

\begin{abstract}
Huber, C, Göpfert, B, Kugler, P, and von Tscharner, V. The effect of sprint and endurance training on EMG signal analysis by wavelets. J Strength Cond Res 24(X): 000-000, 2010-The purpose of this study was to relate the spectral changes of surface electromyograms (EMGs) to training regimes. The EMGs of M. vastus medialis and M. vastus lateralis of 8 female sprint-trained and 7 female endurance-trained athletes (ST and ET athletes) were examined while performing isokinetic knee extension on a dynamometer under 4 different loading conditions (angular velocity and load). The EMG signals were wavelet transformed, and the corresponding spectra were classified using a spherical classification, support vector machines (SVMs) and mean frequencies (MFs). Consistent differences in the EMG spectra between the 2 groups were expected because of the difference in the muscle features resulting from the various training regimes. On average, the ST athletes showed a downshift in the EMG spectra compared with the ET athletes. The spectra of the ST and ET athletes were classifiable by spherical classification and SVM but not by the MF. Thus, the different shapes of the EMG spectra contained the information for the classification. The hypothesis that specific muscle differences caused by various training regimes are consistent and lead to systematic changes in surface EMG spectra was confirmed. With the availability of new apparels, ones with integrated EMG electrodes, a measurement of the EMG will be available to coaches more frequently in the near future. The classification of wavelet transformed EMGs will allow monitoring training-related spectral changes.
\end{abstract}

KEY WoRDS isokinetic knee extension, EMG spectra, wavelet analysis, pattern recognition, classification, apparels

Address correspondence to Cora Huber, cora.huber@unibas.ch.

$0(0) / 1-10$

Journal of Strength and Conditioning Research

(C) 2010 National Strength and Conditioning Association

\section{INTRODUCTION}

$\mathrm{M}$ uscle properties are known to change with training. For example, properties such as diameter and fiber-type distribution do change (27). In a study on ET athletes such as marathon runners, the relative fiber type I percentage was higher than in ST athletes (22). A high-velocity isokinetic strength training results in an increase of the cross-sectional area of the elbow flexors (25), and marathon training increased the cross-sectional area of M. gastrocnemius lateralis (27). These changes of muscle properties are reflected in the spectra of an electromyogram (EMG). A study of Wakeling et al. (34) on fish showed that fast twitch fibers generally have a higher mean frequency (MF) than do slow twitch fibers. Von Tscharner and Nigg (33) concluded that the EMG spectra, among other aspects, reflected the task-specific selection of muscle fibers. For instance, the muscle tuning of the M. tibialis anterior before heel strike resulted in the expression of higher frequencies in the EMG signal than those observed after heel strike (32). One can therefore expect that training by using fast or slow movements could affect the distribution of muscle properties that generate high and low frequency components in the EMG spectra.

There are 2 questions that are of interest: First, "Can one use the spectra to assess whether an athlete did speed or endurance training?" and second, "What can one deduce from the spectra about the muscle properties?" The present study primarily addresses the first question, because this may lead to practical applications such as following training results of athletes or assessing their predisposition. However, the second question may yield further insight in a controversial interpretation of EMG spectra. The controversial interpretation has recently been debated in a point counterpoint article $(7,33)$. The authors summarized the properties of the muscles that generate and affect the EMG spectra. The point was "Spectral properties of the surface EMG can characterize motor unit (MU) recruitment strategies and muscle fiber type" (33). According to the "point," the spectral differences should allow the classification of speed or endurance-trained athletes. 
To interpret the frequency changes, one has to be aware that the spectrum of an EMG is primarily determined by the shapes of the MU action potentials (MUAPs) as generated along the muscle. A MUAP in turn translates to a shape in time (MUAPt), which is observed by the measuring device at a fixed position. The translation from MUAPs to MUAPt results from the conduction velocity (CV) (18). An interference EMG is generated by a superposition of the different MUAPts. The type of training may change many of the properties of a muscle, especially properties that change the size of the muscle and its composition and neuromuscular control. However, most frequently, discussions were boiled down to changes of $\mathrm{CV}$ that then alter the MF of the spectra. The CV is influenced by the type (8), proportion (22), and the cross-sectional area of the muscle fiber (17) among other factors. However Troni et al. (28) showed that the CVs were almost normally distributed and form a single peak and thus contained not much of a detailed structure. One has therefore to consider that fiber type, proportion, and cross sectional area also change the shape of the MUAP and thus the spectra. Farina et al. (9) commented on various reasons for changing the MF of the EMG spectra. In a study on the M. abductor pollicis brevis, it was clearly shown that besides changes of conduction velocities also the shape of the spectra changed with fatigue (1) thus indicating changes in the shape of the MUAP. One of the factors that strongly determines the shape and the size of the MUAP is the endplate distribution in the innervation zone. The area of the innervation zone (26) represents a primary component affecting the shape of the MUAP and thus the spectra. All the features of the muscle may somehow change during the training of the athletes. One can therefore expect that the spectra of speed and endurance-trained athletes will be of different shapes. The question remains whether these different shapes are consistently different and allow the assessment of the training status.

In many studies, the assessment of spectral differences was reduced to measuring the MF (e.g., [13,21]). However, different spectral shapes resulting from altered MUAP cannot be represented by a single variable. These spectral differences can be expected to be very subtle if the groups comprise noninjured athletes. To be able to discuss the spectral differences recorded for 2 different groups, the EMG spectra have to be characteristic for the training of the groups. A pattern recognition method has to be applied to the spectra to decide whether they correlate with a training regime. Nowadays, very complex pattern recognition methods are available $(2,5,6,10)$; one of them, the spherical classification was previously applied to EMG signals $(16,30)$. Classification is not necessarily possible by using one muscle at a time. Muscles work in concert, and therefore, multiple muscles were included for classification purposes (31). As the theory of classification evolves, the selected methods have to be carefully evaluated against one another. In some cases, a projection to higher dimensional spaces lead to a linear classification of the samples, whereas in other cases, a reduction of dimensionality is more appropriate. For the classification of EMGs, some propositions have been made (19).

The purpose of this study was to test the hypothesis that the spectra of EMG recordings of female athletes that were trained for sprinting (ST athletes) or endurance tasks (ET athletes) were sufficiently different to allow assigning an athlete to one of the groups. Specifically, the spectral differences in M. vastus lateralis (VL) and M. vastus medialis (VM) muscles resulting from the different training regimes were of interest for the 2 different running tasks. It was hypothesized that the differences become classifiable by using nonlinearly scaled wavelet analysis of the EMGs (29) combined with a spherical classification method (30) or a support vector machine (SVM) (3). Both methods have been recently developed and were to our knowledge not previously applied to EMG signals. They are important because new apparels with integrated EMG electrodes become available to athletes; however, the analysis methods that yield relevant information for coaches have not yet been sufficiently developed. It is important to validate and discuss new analysis methods that will enable the coaches to deduce from EMGs whether the training resulted in an EMG that is typical for the training regimes.

\section{Methods}

\section{Experimental Approach to the Problem}

This study was designed to address the question of how endurance and sprint training affect the muscle activity during knee extensions.

\section{Subjects}

Athletes who trained by fast movements, for example, sprinters and bobsleigh pushers, were referred to as ST athletes, whereas marathon runners and triathlon athletes were referred to as ET athletes. Eight female ST athletes (mean $\pm S D$ age $=24.1 \pm 3.5$ years; mean $\pm S D$ height $=$ $170.8 \pm 7.2 \mathrm{~cm}$; mean $\pm S D$ mass $=65.7 \pm 8.8 \mathrm{~kg})$ and 7 female ET athletes (mean $\pm S D$ age $=26.7 \pm 5.0$ years; mean $\pm S D$ height $=169.9 \pm 4.0 \mathrm{~cm}$; mean $\pm S D$ mass $=$ $57.0 \pm 5.4 \mathrm{~kg}$ ) volunteered for this study. The ST and ET athletes were in the Swiss national top 15 of their distance and age group and were training for the winter season competition (December 2006 to early March 2007). They all have at least 4 years of experience in participating in competitions. The ST group consists of athletes from track and field (100 m and 100-m hurdles) and athletes of the Swiss national world cup team in bobsleigh. The ET athletes were either runners on the track (distances longer than $1,500 \mathrm{~m}$ ) or road runners (triathlon athletes and marathon runners). The exclusion criterion was that the athletes had no previous knee or leg injury.

The investigation was approved by the official Ethics Committee of Basel (EKBB), Basel, Switzerland. Subjects were informed of the experimental risks and signed an informed consent document before the investigation. All 
athletes indicated their age and dominant leg side, and their height and weight were measured.

\section{Procedures}

The measurements were made on a hydraulic isokinetic dynamometer of type Cybex Orthotron KT 2 (Cybex, Medway, MA, USA). The athletes were seated with flexed legs on the dynamometer with a hip flexion angle of $100^{\circ}$. Trunk and both thighs were fixed with belts to minimize muscular compensation and evasive moments. The 2 knee adapters were fixed at half the distance from the fibula head to the lateral maleoli.

The athletes executed knee extensions under 4 different conditions: (a) velocity $>105^{\circ} \cdot \mathrm{s}^{-1}$ and load $<27 \mathrm{~N} \cdot \mathrm{m}$, (b) velocity $>105^{\circ} \cdot \mathrm{s}^{-1}$ and load $>50 \mathrm{~N} \cdot \mathrm{m}$, (c) velocity $<$ $32^{\circ} \cdot \mathrm{s}^{-1}$ and load $<27 \mathrm{~N} \cdot \mathrm{m}$, and (d) velocity $<32^{\circ} \cdot \mathrm{s}^{-1}$ and

T1 load $>50 \mathrm{~N} \cdot \mathrm{m}$ (Table 1 ). For each condition, the subjects performed 8 trials, starting with condition 1 and finishing with condition 4 , through a movement range of $90^{\circ}$ knee extension (start and end knee angles were $90^{\circ}$ and $0^{\circ}$, respectively). Bad trials were repeated if the load output and angular velocity was not within the range of the requested condition. One ET athlete was unable to reach the load level under the given angular velocity in condition 2 ; therefore, the ET group consists only of 6 ET athletes that have fulfilled condition 2. For further analysis, the 5 trials closest to the median value (Table 1 ) were used, thus minimizing the effects of outliers. The knee extension from $60^{\circ}$ to $30^{\circ}$ was employed.

Kinematic and kinetic signals of the knee were simultaneously measured by using the following equipment: Goniometer $\left(\mathrm{LOB}^{2}\right.$, Basel, Switzerland) for measuring knee angle over time, Acceleration sensor (Biovision, Wehrheim, Germany) for controlling dynamometer angle, Torque sensor (Cybex, Medway, MA, USA) for measuring torque over time in the knee and in the dynamometer axe, respectively. The kinematic and kinetic signals were smoothed with a $50-\mathrm{Hz}$ high-pass Butterworth filter (fourth order) and resampled at $500 \mathrm{~Hz}$.

The muscle activity of the VL and the VM were measured on the dominant leg side using an EMG system (Biovision, Wehrheim, Germany). The placement of the bipolar $\mathrm{Ag} / \mathrm{AgCl}$ surface electrodes with a diameter of $10 \mathrm{~mm}$ and an interelectrode distance of $22 \mathrm{~mm}$ (Noraxon USA Inc.,
Scottsdale, AZ, USA) were in accordance with SENIAM recommendations (14). The reference electrode was placed on the tibial tuberosity of the dominant leg. All electrodes were connected to single differential amplifiers with a band path of $10-700 \mathrm{~Hz}$ (Biovision, Wehrheim, Germany). The EMG was sampled at $2,520 \mathrm{~Hz}$ using a DAQ-Card (DAQCard-6036E, National Instruments Corporation, Austin, TX, USA) and saved on a laptop computer.

The EMG signals were submitted to a time-frequency analysis described by von Tscharner (29). This analysis method consists of a filter bank of 14 nonlinearly scaled wavelets (Cauchy wavelets) indexed by j. Each EMG signal was filtered by the 14 wavelets that were characterized by their center frequency $\left(\mathrm{cf}_{j}\right)(7,19,38,62,92,128,170,218$, 272, 331, 395, 457, 542, 624, and Hz). The wavelets were abbreviated as, for example, $w_{457 \mathrm{~Hz}}$ indicating that this wavelet had a center frequency of $457 \mathrm{~Hz}$. The bandwidth of the filter and the time resolution were computed previously (29). The EMG signal was convolved by these wavelets yielding an intensity $p_{j, n}$, where $n$ represents the index for the time points. The intensity of $w_{7 \mathrm{~Hz}}$ was not further considered because it is most likely affected by movement artifacts (4). The intensity $p_{j, n}$ is proportional to the EMG power within the frequency band. The intensities $p_{j, n}$ for one specific $n$ are called a wavelet spectrum, which is equivalent to a power spectrum. The magnitude of an intensity pattern is the square root of the sum of all intensities squared. During the wavelet transformation, the data were resampled at $500 \mathrm{~Hz}$. The results $p_{j, n}$ were visualized as EMG intensity patterns by showing contour plots where the abscissa represents time, the ordinate the frequency, and the contours and gray shades the intensity.

Electromyogram wavelet spectra were used to monitor changes in the MU properties resulting from the training. To obtain representative spectra, an averaging procedure was used.

The average of the wavelet spectra obtained for the movement range of $60-30^{\circ}$ knee extension was called the spectral vector (s_vector) of an intensity pattern. All s_vectors were normalized to total power of 1 by dividing the s_vector through the sum of its components. For each athlete indexed by $\mathrm{k}$ and condition, a mean_s_vector was computed by building the average of the 5 s_vectors. To use the

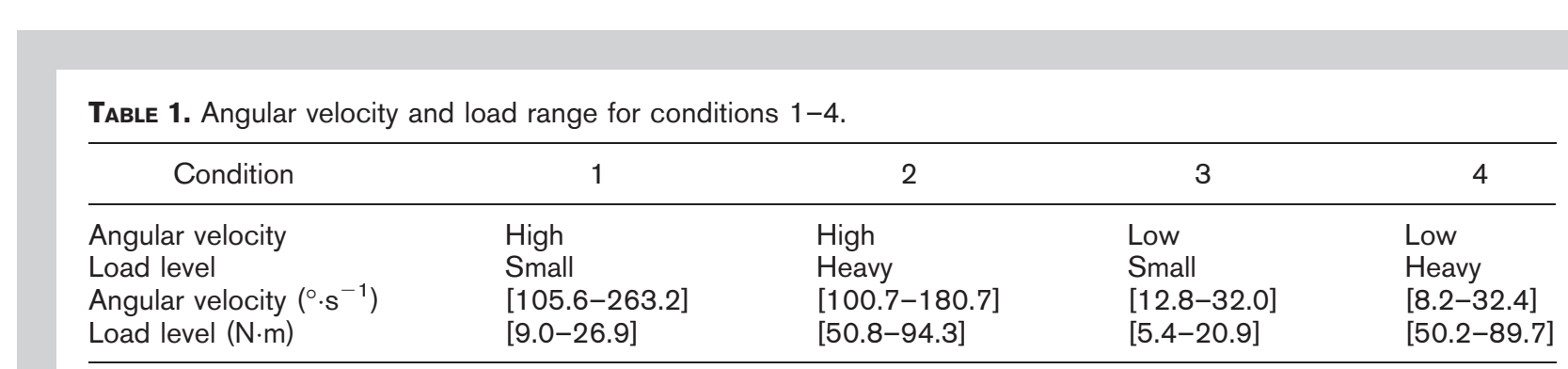


mean_s_vectors for classification purposes, only a limited range containing the information is required, and the data have to be appropriately rearranged. The power of the mean_s_vectors of $w_{\text {start } \mathrm{Hz}}$ up to $w_{\text {end } \mathrm{Hz}}$ of the VL and VM were stacked on top of each other to form one long vector. The range of frequencies (start to end) was selected based on the results shown later. These vectors were arranged as columns in a matrix $W_{\mathrm{ST}}$ and $W_{\mathrm{ET}}$, respectively.

The group mean across the subjects was computed for $W_{\mathrm{ST}}$ and $W_{\mathrm{ET}}$. The group mean spectra were visually inspected. Two spectra were considered to have different shapes if they could not be brought to superposition by normalization and rescaling of the frequency axis. There were 4 matrices for $W_{\mathrm{ST}}$ and $W_{\mathrm{ET}}$ representing the spectra of the 4 conditions. These matrices were used as input to the classification methods (spherical classification, SVM classification and MF classification).

Spherical classification of the wavelet transformed EMG intensity pattern was presented previously by von Tscharner (30). The spherical classification was described in general by Fukunaga (10). In this study, the spherical classification of von Tscharner (30) was adapted to the EMG spectra, whereas the vector ' $g$ ' represents the distances of a mean s_spectra to the midpoint of the shell of each of the spheres. If $g_{\mathrm{ST}, \mathrm{k}}$ is larger than $g_{\mathrm{ET}, \mathrm{k}}$, then athlete $\mathrm{k}$ belongs to the ST group, otherwise to the ET group. This assignment criterion was used to assess separability and classification. A crossvalidation was done by using a leave-one-out method $(10,30)$. In the leave-one-out method, one subject, the one left out, was used as a new test subject and eliminated from the $W_{\mathrm{ST}}$ and $W_{\mathrm{ET}}$. The separability was computed for the subjects in the reduced $W_{\mathrm{ST}}$ and $W_{\mathrm{ET}}$, whereas the classification of the test subject was performed using the spheres obtained from the reduced $W_{\mathrm{ST}}$ and $W_{\mathrm{ET}}$. This procedure was repeated using each subject once as a test subject. Thus, for each subject, one gets to know (a) whether it was correctly assigned to its group (classifiable) and (b) the separability of the remaining subjects that were used as controls. The final result of the crossvalidation was (a) the average separability of each of these tests and (b) the crossvalidation rate (number of classifiable test subjects divided by the total number of subjects). To visualize the spherical classification process, the distances $\left|g_{\mathrm{ST}, \mathrm{k}}\right|$ and $\left|g_{\mathrm{ET}, \mathrm{k}}\right|$ were plotted logarithmically against each other. A $45^{\circ}$ diagonal line separates the 2 groups. If there is $100 \%$ separability, the 2 groups appeared on each side of the diagonal line.

Support vector machines have attracted great attention over the last decade (11). The SVM software was downloaded as freeware from the internet (3). In the SVM, one still has to decide which kernel to use, and there is an adjustable parameter $C$ that controls the performance of classification. We selected a linear kernel and tested $C$ values between 1 and 1,000. A $C$ value of 50 was selected as an educated guess by an experienced user. The $W$ matrices were used as input to the SVM. The same crossvalidation using the leave-one-out method described above was used. The results were, as for the spherical classification, the average separability and the crossvalidation rate.

In the more classical EMG analysis, the MF was used as a measure of spectral differences. The group MF for the different muscles was computed for the classical comparisons. The purpose of this study, however, requires a classification of an athlete as belonging to the ST or ET group. Therefore, the following classification method based on MF was used. The MFs for the group mean of $W_{\mathrm{ST}}$ and $W_{\mathrm{ET}}$ were computed for each condition as a weighted average of the center frequencies. Thus $2 \mathrm{MFs}$ were obtained, one for the $\mathrm{VL}, \mathrm{MF}_{\mathrm{VL}}$, the other for $\mathrm{VM}, \mathrm{MF}_{\mathrm{VM}}$. A subject was deemed correctly assigned to its group if both, $\mathrm{MF}_{\mathrm{VL}}$ and $\mathrm{MF}_{\mathrm{VM}}$ were closer to their respective group mean. Some subjects were not assignable because both MFs were not simultaneously closer to their respective means. A crossvalidation using the leaveone-out method was used to compute the crossvalidation rate of the assignable subjects and an overall crossvalidation rate. In this case, the group mean of $\mathrm{MF}_{\mathrm{VL}}$ and $\mathrm{MF}_{\mathrm{VM}}$ of the control subjects did not contain the $\mathrm{MF}_{\mathrm{VL}}$ and $\mathrm{MF}_{\mathrm{VM}}$ of the subject that was left out to be tested. The result of the crossvalidation for MF were (a) the rounded average assignable control subjects, (b) the average separability (correctly assigned control subjects divided by the assignable control subjects), (c) the assignable test subjects (d), the crossvalidation rate (classifiable test subjects divided by average assignable subjects), and (e) the overall crossvalidation rate (classifiable test subjects divided by number of subjects). The overall crossvalidation rate corresponds to the product of the prior probability of a subject being assignable with the probability being assigned to the correct group (crossvalidation rate).

All signal processing was performed using programs written in the Matlab programming software (MathWorks, Version 7.1).

\section{Statistical Analyses}

Statistical analyses typical for pattern recognition methods were applied. In a high-dimensional vector space, one can often obtain perfect separability $(100 \%)$ by a discriminant. However, a new subject is not necessarily assigned to the correct group by this discriminant. A correctly assigned new subject was called classifiable. A leave-one-out crossvalidation procedure (10) was used to obtain the crossvalidation rate (classifiable subjects divided by total subjects) that indicates the probability of correctly assigning an unknown subject to the ST or ET group. If the assignment would be random, one would obtain a $50 \%$ crossvalidation rate. A binomial test with equal probability for the 2 conditions (belonging to group \#1 or group \#2) was used to determine the statistical significance of the classifiable subjects and thus of the crossvalidation rate. The computed one sided $p$ value indicated the cumulative probability of getting a number of classifiable subjects greater or equal to the reported classification as a result of random assignments. If the $p$ 
value was below 0.05 , then the hypothesis that the classification was a result caused by random assignments can be rejected and thus the crossvalidation rate was deemed significant. The binomial test was applied to the crossvalidation rate of all 3 classification methods. Whether the mean of the MF of the 2 groups were significantly different was irrelevant for the classification. However, because the MF was one of the most important variables in the past, the group differences for the MF variables were analyzed with an unpaired Student $t$-test with Microsoft Excel 2002 (Microsoft Inc., Redmond, WA, USA).

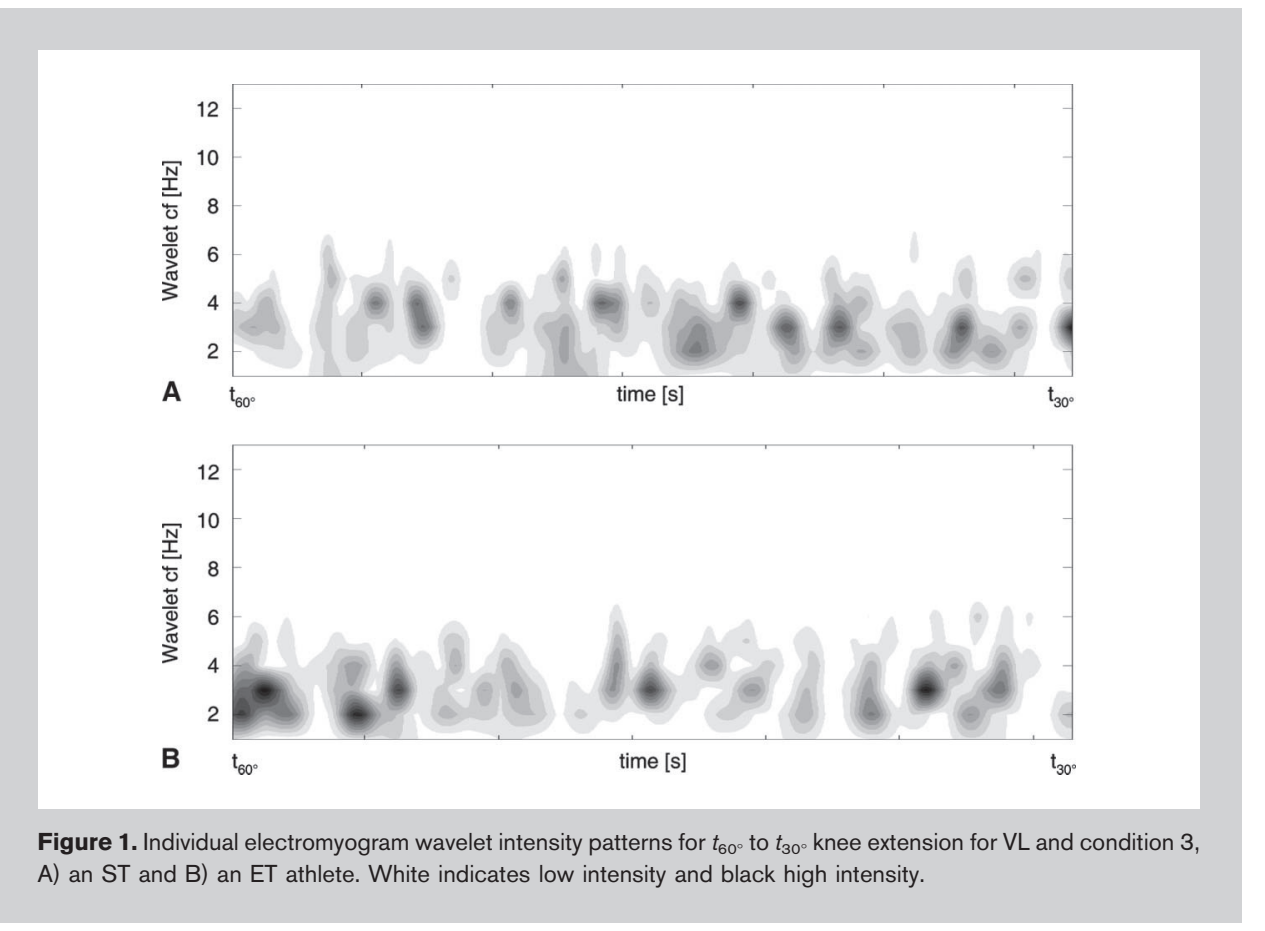

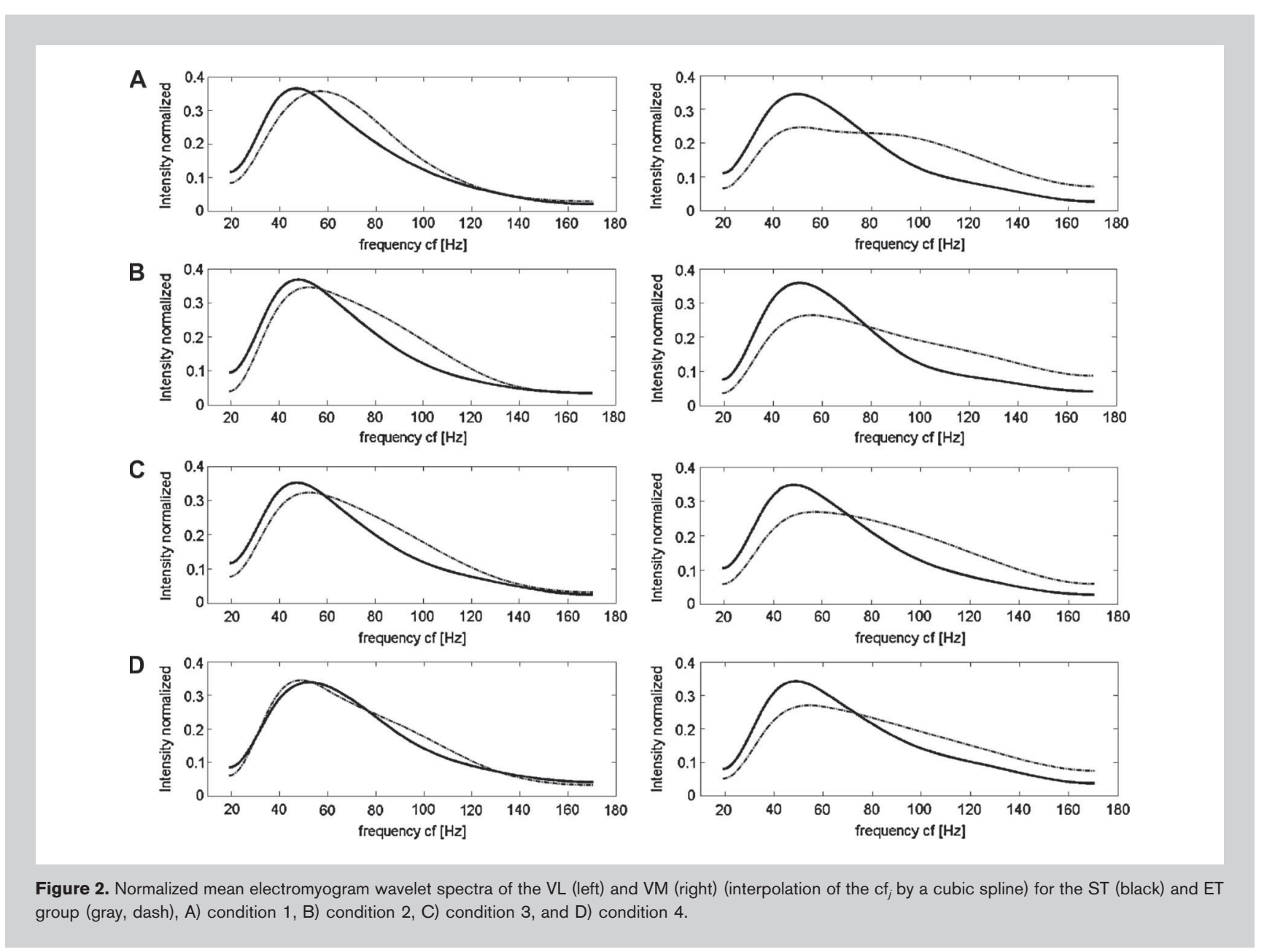


TABLE 2. Mean frequencies for conditions $1-4$. $^{*}$

\begin{tabular}{|c|c|c|c|c|}
\hline Condition & 1 & 2 & 3 & 4 \\
\hline Number of subjects & \multicolumn{4}{|c|}{ M. vastus lateralis (MF (SE) [Hz]) } \\
\hline ST & $62.86(3.31)$ & $67.11(5.08)$ & $66.80(3.32)$ & $72.66(4.51)$ \\
\hline ET & $69.03(2.40)$ & $75.23(4.82)$ & $75.27(2.65)$ & $73.04(1.47)$ \\
\hline$p$ value of $t$-test & \multicolumn{3}{|c|}{ M. vastus medialis (MF (SE) [Hz]) } & 0.94 \\
\hline ST & $67.65(4.11)$ & $72.86(6.53)$ & $69.72(3.28)$ & $73.58(5.92)$ \\
\hline ET & $89.19(3.15)$ & $96.20(4.17)$ & $88.92(3.56)$ & $89.65(2.50)$ \\
\hline$p$ value of $t$-test & $0.001 \dagger$ & $0.01 \dagger$ & $0.002 \uparrow$ & $0.03 \dagger$ \\
\hline
\end{tabular}

${ }^{\star} \mathrm{ET}=$ endurance-trained; MF = mean frequency; SE = standard error in parentheses; ST = sprint trained.

$\uparrow$ Significant different mean frequency between the 2 groups $(p<0.05)$.

\section{Results}

Typical intensity patterns for ST athletes and ET athletes are F1 shown in Figures 1A, B, respectively. These patterns were recorded for the movement range of $60^{\circ}-30^{\circ}$ knee extension for VL. These examples were recorded for condition 3, thus representing low angular velocity and small load. The patterns showed a series of muscle activities that were spread over the duration of the movement. They seem to be arranged in regular time intervals of about 0.08 seconds. These typical repetitive muscle activities can be found in both muscles of all subjects, irrespective of the conditions. These patterns were so variable that one could not visually assign them to one or the other condition. The spectra in the range $60^{\circ}-50^{\circ}$, and in the range $40^{\circ}-30^{\circ}$ knee extension indicated that there was a slight frequency increase with the knee angle (results not shown). The increases are small enough that the following analysis could be limited to the mean spectra recorded over the full range of $60^{\circ}-30^{\circ}$ knee extension. These spectra were normalized to a total power of 1 .

The averaged normalized EMG wavelet spectra of the $\mathrm{VL}$ and VM were shown in Figures 2A-D for each condition of the ST and the ET group, respectively. Visual assessment of the spectra between the 2 groups showed that significant power was only observed up to $w_{170 \mathrm{~Hz}}$. In general, although not for spectra in Figure 2A for VL, the spectra of both groups revealed a different shape. Higher proportions of the lower frequency components can be seen for the ST group. The detailed analysis showed that the average $\mathrm{MF}_{\mathrm{VL}}$ was significantly higher in the ET group than in the ST group, whereas the average $\mathrm{MF}_{\mathrm{VM}}$ 
showed the same trend but was not significantly different

T2 (Table 2).

Whether the visually observed differences in the shape of the spectra contained enough information to decide about the training status of the athletes had to be assessed numerically. The 3 possible classification methods, spherical classification, SVM, and the MF classification yielded the following results.

The separability of the spectra obtained by the spherical classification method was shown for F3 condition 1 in Figure 3. The ST subjects (dots) have a smaller distance from the shell center of their group and are therefore located closer to the ordinate. The ET subjects (triangle) have a smaller distance from the shell center of their group and are therefore located closer to the abscissa. The $45^{\circ}$ diagonal line represents the line separating the 2 groups.

The spherical classification indicated that the spectra of the athletes of the 2 groups were over $83 \%$ separable and over 73\% classifiable for unknown T3 subjects as shown in Table 3. According to a binomial test, there was a significant classification as indicated by the $p$ values of the crossvalidation rate for conditions $1-3$. For condition 4, there were 11 classifiable subjects which was a similar absolute number than for the other conditions. According to Fukunaga (10), the spherical classification does not necessarily lead to the absolute best classification. Therefore, the results had to be compared using mathematically more demanding methods.

The SVM using 4 eigenvalues indicated that the spectra of the athletes of the 2 groups were $100 \%$ separable as shown in

Table 4. The crossvalidation rate was between $71 \%$ and $93 \%$ for the classification of unknown subjects. The results of the SVM were for all conditions except for condition 2 (using 4 eigenvalues). out method. out method. leave-one-out method. higher than for the spherical classification. Reanalysis of the data without subject 14 altered the crossvalidation rate of condition 2 from $71 \%$ to $93 \%$. Thus, subject 14 most likely represented an outlier.

The classification based on MFs was more cumbersome because only a limited number of subjects could be assigned to

TABLE 3. Results of the spherical separation and classification for conditions 1-4

\begin{tabular}{lllll}
\hline \multicolumn{1}{c}{ Condition } & 1 & 2 & 3 & 4 \\
\hline Number of subjects & 15 & 14 & 15 & 15 \\
Average separability (\%) & 90.5 & 87.4 & 99.5 & 83.3 \\
Classifiable & 12 & 11 & 12 & 11 \\
Crossvalidation rate (\%) & $80^{*}$ & $78.6^{*}$ & $80^{*}$ & 73.3 \\
$p$ Value of crossvalidation rate & 0.02 & 0.03 & 0.02 & 0.06 \\
\hline
\end{tabular}

*Significant crossvalidation rate $(p<0.05)$. The classification was done by the leave-one-

TABLE 4. Results of the support vector machine classification for conditions 1-4.

\begin{tabular}{lcccc}
\hline \multicolumn{1}{c}{ Condition } & 1 & 2 & 3 & 4 \\
\hline Number of subjects & 15 & 14 & 15 & 15 \\
Average separability (\%) & 100 & 100 & 100 & 100 \\
Classifiable & 14 & 10 & 14 & 14 \\
Crossvalidation rate (\%) & $93.3^{*}$ & 71.4 & $93.3^{*}$ & $93.3^{*}$ \\
$p$ Value of crossvalidation rate & 0.0005 & 0.09 & 0.0005 & 0.0005 \\
\hline
\end{tabular}

*Significant crossvalidation rate $(p<0.05)$. The classification was done by the leave-one-

TABLE 5. Results of the mean frequency classification for conditions 1-4.

\begin{tabular}{lcccc}
\hline \multicolumn{1}{c}{ Condition } & 1 & 2 & 3 & 4 \\
\hline Number of subjects & 15 & 14 & 15 & 15 \\
Average assignable (rounded) & 11 & 10 & 13 & 8 \\
Average separability (\%) & 84 & 90 & 90 & 85 \\
Assignable test subjects & 10 & 10 & 13 & 4 \\
Classifiable test subjects & 8 & 9 & 11 & 3 \\
Crossvalidation rate (\%) & 73 & $90^{*}$ & $85^{\star}$ & 38 \\
p Value of crossvalidation rate & 0.11 & 0.01 & 0.01 & 0.85 \\
Overall crossvalidation rate (\%) & 53 & 64 & $73^{\dagger}$ & 20 \\
$p$ Value of overall crossvalidation rate & 0.50 & 0.21 & 0.06 & 0.99 \\
\hline
\end{tabular}

*Significant crossvalidation rate.

$\dagger$ Significant overall crossvalidation rate $(p<0.05)$. The classification was done by the 
T5 one or the other group (Table 5). In contrast to the spherical classification and the SVM classification, there was onequarter of the cases (subject and condition) not assignable. The analyses of those subjects that were assignable yielded a crossvalidation rate that was significant for 2 conditions only. However, the overall crossvalidation rate indicated that the subjects that were either not assignable or miss-assigned were very large. Considering the $p$ values of the overall crossvalidation rate, an assignment of an unknown subject based on MF was not reliable.

\section{Discussion}

Our results confirmed the hypothesis that specific muscle differences caused by various training regimes in sprinting and running are reflected in systematic differences in surface EMG spectra when a filter bank of wavelets was used to extract them. These differences were sufficiently distinct to allow detecting whether an athlete has been trained for a sprinting or an endurance task, irrespective of the details of the training regime. However, the isometric EMG measurements published by various researchers (e.g., [21]) could not explain the spectral changes that we observed during dynamic movements. Different kinds of dynamic movements do change the muscle features as discussed below. Shepstone et al. (25) measured larger diameter in muscle fibers after a fast strength training for both the type I and type II fibers. According to classical EMG theory, larger fiber diameter should lead to higher CV (17) and shifts to higher frequencies were experimentally confirmed (12). These results were obtained for isometric contractions using a force that corresponded to $70 \%$ of maximal voluntary contraction. Based on these 2 relationships, increase of fiber diameter for $\mathrm{ST}$ athletes and increase in $\mathrm{CV}$, one would expect that ST subjects would have higher MFs in their spectra. However, our results showed on average lower MFs for the ST subjects than for the ET subjects (Table 2). According to these results, one would be inclined to conclude that the usual explanation of the relationship between fiber type, $\mathrm{CV}$, and frequency are not sufficiently understood to explain the results. However, the present results are in accordance with studies that showed that even though the $\mathrm{CV}$ did change, the MF did not (10). In some cases, the MF was independent of the fiber diameter (34). One might argue that the basic relationships were usually measured during nondynamic loading conditions. It could therefore be that sprint training has increased the ability to synchronize the MUAP, thus decreasing the MF. It is not known under what circumstances the synchronization increases and should be considered to explain the effect. As stated previously, the riddle will only be resolvable by simultaneously measuring the EMG spectra and the $\mathrm{CV}(7,33)$. These inconsistencies may suggest that the spectra may not be characteristic for the trained task. However, our results clearly showed that the spectra were sufficiently consistent to be able in most cases to correctly assign an unknown EMG spectrum to the ST or ET group by using a spherical classification or SVM classification. For a correct classification, the information contained in the whole spectra was relevant, whereas the MF contained not enough information. The information was therefore contained in the shape of the spectra. These shapes were visibly different for the 2 groups, as shown in Figure 2, and the differences cannot be explained by a change in CV only. The detection of differences in shape required using pattern recognition methods. The advantage of using the spherical classification was its simplicity. The advantage of the SVM, as long as one only considers a linear kernel and an appropriate value of $C$, is that it might in future also reveal which part of the spectra contained the information required for the classification.

The shape of the spectra depends on the recruitment of the selected MUs (central control) and on the peripheral buildup of the interference EMG. A high correlation of MUs can also be described as synchronization (15). Semmler and Nordstrom (24) were able to show that strength trained subjects had a greater degree of synchronization of MU than untrained subjects. The higher the synchronization of MUs, the more the muscle fibers became activated. This helped in performing a fast force production (23), such as that needed in sprinting, where muscles have to work in concert. Synchronization of MU "leads to an absolute increase of power in the EMG spectra at lower frequencies, and to a relative decrease of power at high frequencies" (35). Synchronization results in a downshift of the MF (15). These findings are in contrast to the increase in $\mathrm{MF}$ during isometric contractions observed for many years (18). It is often supposed that during isometric contractions performed at maximum voluntary contraction, all muscle fibers will be activated. However, in dynamic conditions, spectral changes may result from specifically selected muscle fibers during the actual movement of the performing athlete. The selected muscle fibers strongly depend on their availability. Muscle biopsies indicate which fibers are available and how the proportion changes over the training period (27). They do not reflect the immediate selection of fibers. Although we may not be able to explain and fully understand the details behind the spectra, this work clearly shows that the spectra change in a systematic way and therefore contain practical reliable information about the training of the athlete. One can therefore conclude that an EMG spectrum recorded during a dynamic sports movement yields its own information about the muscle condition in addition to the information obtained by EMG spectra recorded for isometric contractions or muscle biopsies. The analysis of the condition of muscles will become a multifactorial task.

\section{Practical Applications}

The coaches would always like to know whether an athlete's muscle response is in accordance to the chosen training regime and whether an athlete has reached the optimal muscular adaptation. The athletes would like to optimally 
tune their muscles for the competition to gain an advantage over the other competing athletes because it is known that the tuning of muscular system can be actively influenced (20). With the availability of new apparels, ones with integrated EMG electrodes, a measurement of the EMG will be available to coaches more frequently in the near future. This work shows that the classical analysis of an EMG based on MFs is not able to assess the training status of an athlete. It was therefore important to develop, validate, and discuss analysis methods. The new wavelet based methods that are currently being tested represent such analysis methods. In combination with the newest pattern recognition methods, it allows the classification of the wavelet EMG spectra that result from different trainings regimes and levels.

The interpretation of the results indicates that ST athletes may improve the ability to synchronize their muscle activation. Because of the better synchronization, the movement might be more precise, and therefore, less energy is used for adjustments. It can be assumed that the training of fast movements and synchronization helps in improving the energy efficiency in normal tasks.

\section{Acknowledgments}

The data analysis has been financially supported by the proMotio Foundation, Basel, Switzerland. Nonadditional financial support was received. We would like to thank the Physiotherapy of the Felix-Platter Hospital, Basel, Switzerland, for using the dynamometer and the Laboratory for Movement Analysis of the University Children Hospital Basel, Switzerland.

\section{REFERENCES}

1. Barandun, M, von Tscharner, V, Meuli-Simmen, C, Bowen, V, and Valderrabano, V. Frequency and conduction velocity analysis of the abductor pollicis brevis muscle during early fatigue. J Electromyogr Kinesiol 19: 65-74, 2009.

2. Bishop, CM. Pattern Recognition and Machine Learning. New York, USA: Springer Science and Business Media, LLC, 2006.

3. Chang, CC and Lin, CJ. LIBSVM: A library for support vector machines. Available at: http://www.csie.ntu.edu.tw/ cjlin/libsvm. Accessed November, 2008.

4. Conforto, S, D'Alessio, T, and Pignatelli, S. Optimal rejection of movement artefacts from myoelectric signals by means of a wavelet filtering procedure. J Electromyogr Kinesiol 9: 47-57, 1999.

5. Cortes, V and Vapnik, V. Support-vector networks. Mach Learn 20: 273-297, 1995.

6. Cristianini, N and Shawe-Taylor, J. Support Vector Machines and Other Kernel-Based Learning Methods. Cambridge, United Kingdom: Cambridge University Press, 2000.

7. Farina, D. Counterpoint: Spectral properties of the surface EMG do not provide information about motor unit recruitment strategies and muscle fiber type. J Appl Physiol 105: 1673-1674, 2008.

8. Farina, D, Ferguson, RA, Macaluso, A, and De Vito, G. Correlation of average muscle fiber conduction velocity measured during cycling exercise with myosin heavy chain composition, lactate threshold, and $\dot{\mathrm{V}}_{2}$ max. J Electromyogr Kinesiol 17: 393-400, 2007.

9. Farina, D, Merletti, R, and Enoka, RM. The extraction of neural strategies from the surface EMG. J Appl Physiol 96: 1486-1495, 2004 .
10. Fukunaga, K. Introduction to Statistical Pattern Recognition. San Diego, CA: Academic Press, 1990.

11. Gardy, JL, Spencer, C, Wang, K, Ester, M, Tusnady, GE, Simon, I, Hua, S, deFays, K, Lambert, C, Nakai, K, and Brinkman, FSC. PSORT-B: Improving protein subcellular localization prediction for Gram-negative bacteria. Nucleic Acids Res 31: 3613-3617, 2003.

12. Gerdle, B, Karlsson, S, Crenshaw, AG, and Fridén, J. The relationship between EMG and muscle morphology throughout sustained static knee extension at two submaximal force levels. Acta Physiol Scand 160: 341-351, 1997.

13. Gerdle, B, Wretling, ML, and Henriksson-Larsén, K. Do the fibretype proportion and the angular velocity influence the mean power frequency of the electromyogram? Acta Physiol Scand 134: 341-346, 1988.

14. Hermens, HJ, Freriks, B, Disselhorst-Klug, C, and Rau, G. Development of recommendations for SEMG sensors and sensor placement procedures. J Electromyogr Kinesiol 10: 361-374, 2000.

15. Jiang, N, Parker, PA, and Englehart, KB. The motor unit innervation process correlation and its effects on EMG applications. Conf Proc IEEE Eng Med Biol Soc 4: 4239-4242, 2005.

16. Jimenez, LO and Landgrebe, DA. Supervised classification in highdimensional space: Geometrical, statistical, and asymptotical properties of multivariate data. IEEE Trans Syst Man Cybern 28: 39-54, 1998

17. Kupa, EJ, Roy, SH, Kandarian, SC, and De Luca, CJ. Effects of muscle fiber type and size on EMG median frequency and conduction velocity. J Appl Physiol 79: 23-32, 1995.

18. Lindstrom, LH and Magnusson, RI. Interpretation of myoelectric power spectra: A model and its applications. Proc IEEE 65: 653-662, 1977.

19. Mulavara, AP, Sastry, KLA, and Verstraete, MC. Frequency characterization of EMG activity during gait. Conf Proc IEEE Eng Med Biol Soc 1215-1216, 1993.

20. Nigg, BM and Wakeling, JM. Impact forces and muscle tuning: A new paradigm. Exerc Sport Sci Rev 29: 37-41, 2001.

21. Rainoldi, A, Gazzoni, M, and Melchiorri, G. Differences in myoelectric manifestations on fatigue in sprinters and long distance runners. Physiol Meas 29: 331-340, 2008.

22. Sadoyama, T, Masuda, T, Miyata, H, and Katsuta, S. Fibre conduction velocity and fibre composition in human vastus lateralis. Eur J Appl Physiol Occup Physiol 57: 767-771, 1988.

23. Semmler, JG. Motor unit synchronization and neuromuscular performance. Exerc Sport Sci Rev 30: 8-14, 2002.

24. Semmler, JG and Nordstrom, MA. Motor unit discharge and force tremor in skill- and strength-trained individuals. Exp Brain Res 119: 27-39, 1998.

25. Shepstone, TN, Tang, JE, Dallaire, S, Schuenke, MD, Staron, RS, and Phillips, SM. Short-term high- vs. low-velocity isokinetic lengthening training results in greater hypertrophy of the elbow flexors in young men. J Appl Physiol 98: 1768-1776, 2005.

26. Stegeman, DF, Blok, JH, Hermens, HJ, and Roeleveld, K. Surface EMG models: Properties and applications. J Electromyogr Kinesiol 10: 313-326, 2000.

27. Trappe, SW, Costill, DL, Fink, WJ, and Pearson, DR. Skeletal muscle characteristics among distance runners: A 20-yr follow-up study. J Appl Physiol 78: 823-829, 1995.

28. Troni, W, Cantello, R, and Rainero, I. Conduction velocity along human muscle fibers in situ. Neurology 33: 1453-1459, 1983.

29. Von Tscharner, V. Intensity analysis in time-frequency space of surface myoelectric signals by wavelets of specified resolution. J Electromyogr Kinesiol 10: 433-445, 2000.

30. Von Tscharner, V. Spherical classification of wavelet transformed EMG intensity patterns. J Electromyogr Kinesiol 2008. [Epub ahead of print]. 
31. Von Tscharner, V and Goepfert, B. Gender dependent EMGs of runners resolved by time/frequency and principal pattern analysis. J Electromyogr Kinesiol 13: 253-272, 2003.

32. Von Tscharner, V, Goepfert, B, and Nigg, BM. Changes in EMG signals fort he muscle tibialis anterior while running barefoot or with shoes resolved by non-linearly scaled wavelets. J Biomech 36: 11691176, 2003.

33. Von Tscharner, V and Nigg, BM. Point: Counterpoint: Spectral properties of the surface EMG can characterize/do not provide information about motor unit recruitment strategies and muscle fiber type. J Appl Physiol 105: 1671-1673, 2008.

34. Wakeling, J, Kaya, M, Temple, GK, Johnston, IA, and Herzog, W. Determining patterns of motor recruitment during locomotion. $J$ Exp Biol 205: 359-369, 2002.

35. Weytjens, J and Steenberghe, D. The effect of motor unit synchronization on the power spectrum of the electromyogram. Biol Cybern 51: 71-77, 1984. 